\title{
LA MOCIÓN DE CENSURA CONSTRUCTIVA. UNA RESPUESTA ALEMANA, Y ESPAÑOLA A LA INESTABILIDAD GUBERNAMENTAL ${ }^{1}$
}

\author{
Ignacio TORRES Muro \\ Departamento de Derecho \\ Constitucional. Facultad de Derecho. \\ Universidad Complutense de Madrid \\ Del Cuerpo de Letrados \\ del Tribunal Constitucional \\ igtorres@ucm.es
}

\section{INTRODUCCIÓN}

No hace falta insistir mucho en estas palabras previas en que, enfrentados a la no sencilla tarea de elaborar una norma suprema, la preocupación principal de los constituyentes españoles de 1977-1978 consistía, por una parte, en no repetir los errores clásicos de la atormentada historia constitucional española ${ }^{2}$, y, por otra, en diseñar un sistema apto para responder a los desafíos propios de una democracia de finales del siglo XX.

En este segundo empeño se miraba al pasado, pero también parecía importante investigar las soluciones constitucionales comparadas, sobre todo de los países europeos, en tanto en cuanto nos proclamábamos afines a los mismos.

Esto hizo que nuestros parlamentarios indagaran en las reglas constitucionales, sobre todo de Alemania, Francia e Italia; países con los que, además, nuestra doctrina jurídica siempre había tenido relaciones fluidas. Uno de los problemas clásicos a resolver era el de la configuración del poder ejecutivo.

${ }^{1}$ La realización de este estudio ha sido posible gracias a los buenos oficios del personal de las bibliotecas de la Facultad de Derecho de la Universidad Complutense y del Tribunal Constitucional. Quede aquí constancia de mi agradecimiento. Aunque no se usan en el mismo demasiadas abreviaturas, no está de más recordar que CE es Constitución Española y LFB es Ley Fundamental de Bonn. El texto se elaboró para un proyecto de libro colectivo que se ha frustrado. En este tiempo cabe reseñar como interesante la aportación de SIMON YARZA, 2015.

2 Para una síntesis de la misma cabe recurrir a Tomás Villaroya, 2012. 
Respecto a una de sus facetas, la manera de regular la jefatura del Estado, poco podían enseñarnos estas naciones, dada la opción española de respetar la existencia de un monarca hereditario al frente de la misma, opción en la que otros modelos (Reino Unido, las monarquías del Benelux o las escandinavas) resultaban más apropiados ${ }^{3}$.

En la tarea de decidir cómo regular las relaciones entre el Parlamento y el ejecutivo sí podían ayudarnos. Más concretamente, en el problema de la posición del gobierno y de su presidente frente a las Cámaras.

Quedó claro desde el primer momento que el gobierno, y, más en concreto, su jefe, debían gozar de la confianza del Parlamento, otorgada mediante un procedimiento de investidura.

No se planteaban entonces las soluciones imaginativas que parte de la doctrina italiana propondría poco después (por ejemplo, la de la elección directa del presidente del consejo $)^{4}$.

Por otra parte, y respecto a las relaciones Gobierno-Cortes, estaba muy presente el rechazo a la tradición española de inestabilidad, que se había iniciado con la crisis del sistema de turnos de partidos de la Restauración a principios del siglo xx y había sido también la regla en la atormentada andadura de la Segunda República. La existencia de una manera de ver sus relaciones con Parlamento y gobierno por parte de ambos jefes del Estado, con la que mostraban sus intenciones de adquirir un protagonismo que resultó contraproducente, había provocado situaciones rechazadas por los constituyentes de 1977.

Alcalá Zamora, por ejemplo, pretendió el mantenimiento del dualismo que había caracterizado nuestro régimen parlamentario en los peores momentos de la Monarquía. Tanto Alfonso XIII ${ }^{5}$ como don Niceto ${ }^{6}$ (apodado, con ironía y con cierta razón, «don Alfonso en rústica») se caracterizaron por ejercer un excesivo intervencionismo.

Hay que reconocer también que facilitó dicha práctica el hecho de tener enfrente unos Parlamentos fragmentados, sin mayorías claras. Dicho intervencionismo provocó una crónica inestabilidad, aprovechada por los críticos españoles de la democracia, sobre todo los que apoyaron el régi-

3 Para la manera en que, a mi modo de ver, deben interpretarse las reglas constitucionales españolas sobre la Corona vid. ToRres Muro, 2009.

${ }^{4}$ La primera vez que se apunta la idea es en GrupPo Di Milano, 1983, t. I: 392 y ss. Mucho después son interesantes las reflexiones sobre el «premierato» contenidas en FrosINI (ed.), 2004.

${ }^{5}$ Vid. sobre la práctica política en su reinado, por ejemplo, MORENo LuZón (ed.), 2003.

${ }^{6}$ Uno de los análisis del problema en Canales Aliende, 1983. 
men del 18 de julio, capitaneado por el general Franco, para desechar la posibilidad de que este sistema político funcionara bien en España y justificar sus delirios de democracia «orgánica», Estado de obras y otras fantasías destinadas a ocultar la cruda realidad de un régimen autoritario.

Los constituyentes no querían, de ningún modo, facilitar la inestabilidad gubernamental, propia de nuestras experiencias democráticas más recientes, y sí establecer un sistema que hiciera posible la combinación de gobiernos «fuertes» con la responsabilidad de los mismos ante los ciudadanos.

Aunque bien es verdad que dicha inestabilidad se debía, en nuestra historia, más a la inadecuada intervención del jefe del Estado (rey o presidente de la República) que a que nuestros Parlamentos tuvieran tendencia a derribar gobiernos.

Confiada, sin embargo, en nuestra nueva norma suprema, la condición de cabeza del sistema constitucional a un monarca sin poderes efectivos, el problema volvía a ser el de que unas Cámaras fragmentadas jugaran, a menudo, a las crisis gubernamentales sin sentido.

Todas estas circunstancias llevaron a descartar dos modelos posibles: el francés de la IV República, ejemplo claro de frecuentes crisis en el ejecutivo, y el italiano de la Constitución de 1947, en el que se reflejaba, como se ha dicho, «el complejo del tirano, en referencia al régimen de Benito Mussolini», de modo que el resultado era «una profunda debilidad constitucional del gobierno y de su jefe con respecto al Parlamento» ${ }^{7}$.

Desde el momento en que la Constitución francesa de 1958 se caracterizaba, en aquella ocasión, por un papel muy disminuido del Parlamento frente al ejecutivo ${ }^{8}$, parece claro que sólo quedaba la «opción alemana». Además, de ésta se sabía que era también un diseño que reaccionaba contra la crónica inestabilidad gubernamental de la experiencia democrática durante la República de Weimar'9 . Puestos los ojos en la Ley Fundamental de Bonn ${ }^{10}$, la regulación de las relaciones del ejecutivo con el Parlamento en España (investidura, moción de censura, moción de confianza, instru-

\footnotetext{
7 Vid., por todos, Pasquino, 2012. La cita en p. 308.

${ }^{8}$ La mejor síntesis del problema en ese momento la hizo, a mi juicio, un autor italiano. Vid. Pegoraro, 1983.

9 Repasa la historia de la Constitución de Weimar Gusy, 1997. Sobre el gobierno y su evolución bajo la vigencia de ese texto vid. pp. 131 y ss.

${ }^{10}$ Una breve, pero enjundiosa, reflexión sobre la influencia de la Ley Fundamental de Bonn en el constitucionalismo español del retorno al Estado democrático de Derecho a finales del siglo XX (Constitución de 1978 y desarrollos posteriores) en CRUZ Villalón, 2011. Sobre la importación de la moción de censura vid. p. 54.
} 
mentos de control, etc.) debe mucho a aquélla, con la importante salvedad del instituto de la disolución ${ }^{11}$, en el que no se importaron las cautelas alemanas y se optó por el modelo, que era el propio de la Gran Bretaña de entonces ${ }^{12}$, de dejarla en manos del jefe del gobierno (art. $115 \mathrm{CE}$ ), acabando por configurar una Kanzlerdemokratie (democracia de canciller) ${ }^{13}$ aún más fuerte que la de su modelo germánico.

Trazado este panorama general, podemos centrarnos ya en uno de los aspectos comunes a Alemania y España: el de cómo se ha regulado la posibilidad, propia de todo régimen parlamentario, de que el Parlamento retire su confianza al jefe del gobierno.

\section{RASGOS PRINCIPALES DE LA REGULACIÓN DE LAS MOCIONES DE CENSURA CONSTRUCTIVAS EN ALEMANIA Y ESPAÑA}

No tendría sentido que pretendiéramos hacer una exposición exhaustiva del régimen de la moción de censura constructiva en nuestros dos países. Dicha tarea ha sido ya abordada por otros con acierto ${ }^{14}$. Lo que pretendemos es ir al núcleo del problema.

En primer lugar conviene insistir en la idea de que ambas naciones se enfrentaban, después del nazismo (Alemania) y tras el régimen del 18 de

${ }^{11}$ En castellano, sobre la disolución en Alemania, comentando las sentencias más llamativas al respecto del Bundesverfassungsgericht, MArTínez Sempere, 1984, y SAiz MoreNO, 1983.

${ }^{12}$ La visión clásica del problema de la disolución en Inglaterra la recoge MARTínEz SEMPERE, 1998. Los cambios más recientes (Fixed Term Parliaments Act de 2011) se comentan en LEYLAND, 2012: 160.

${ }_{13}$ Es una expresión de uso corriente en la doctrina alemana. Dos ejemplos, en manuales clásicos, Maunz y ZipPelius, 1991: 291, y Hesse: 1995: 269.

${ }^{14}$ La bibliografía española sobre el tema de la responsabilidad ante el Parlamento y las mociones de censura es bastante amplia. Por ejemplo, y entre los libros, pueden citarse: Mellado Prado, 1988, que examina la responsabilidad política (principio e instrumentos) en Alemania (Weimar y Ley Fundamental de Bonn), Francia (gaullismo) y España (historia y Constitución de 1978); VIRGALA ForURIA, 1988, que narra la historia española, el modelo alemán y nuestro régimen actual en los niveles nacional y autonómico; SÁNCHEZ DE Dios, 1992, que es un brillante ejercicio de «ciencia política comparada», deteniéndose en las realidades británica, francesa, italiana, alemana y española, y, por fin, ELÍ́As MÉNDEZ, 2005, que se ocupa de los casos alemán y español en los niveles nacional y federado o autonómico. Antes de todos estos desarrollos más ambiciosos contábamos con otra obra más breve, pero que apuntó la mayoría de los problemas. Nos referimos a Montero Gibert y García Morillo, 1984. Por fin cabe hacer referencia a una curiosidad: el breve artículo, en alemán, del lamentablemente fallecido profesor González Encinar. Vid. GonZÁLEz EnCINAR, 1988. 
julio (España), con la necesidad de reconstruir un régimen democrático, blindado frente a los errores en los que se había incurrido en las respectivas historias constitucionales (Weimar, crisis de la Restauración y Segunda República), especialmente en el asunto de las relaciones Gobierno-Parlamento, y el modo de regular la retirada de confianza de este último en el primero, como es propio de un régimen, en principio, parlamentario. La pesadilla a evitar era la inestabilidad que había caracterizado a los periodos a los que me he referido.

Tanto la Constitución de Weimar de 1919 como la española de 1931 previeron en los artículos correspondientes (arts. 54 en Alemania ${ }^{15}$ y 64 en la Constitución de $1931^{16}$ ) unos sistemas de censura que no fueron operativos, también porque sus Parlamentos estaban claramente fragmentados.

Nos encontramos, en las dos normas supremas, con un intento de regular la retirada de la confianza por parte del Parlamento al gobierno, que estuvo, hasta entonces, en el parlamentarismo clásico, poco sometido a normas estrictas. Una situación que se entendía que era necesario cambiar. De ahí la previsión de que la censura sea por «un acuerdo expreso», como dice el art. 54 de la Constitución alemana.

En el caso español, las reglas son más estrictas, puesto que se habla, en el art. 64 del texto de 1931, de una «censura [...] motivada y por escrito» que apoyen «cincuenta diputados en posesión del cargo», con un periodo de enfriamiento de cinco días, y que deberá ser aprobada «por la mayoría absoluta», sin la cual «no se considerará obligado a dimitir el gobierno ni el ninistro».

Como vemos, había normas claras, pero la novedad decisiva de las futuras regulaciones (que la aprobación de la censura implica no sólo la caída del canciller/presidente del gobierno, sino su sustitución automática por otro) ya es cosa de los textos constitucionales de la segunda posguerra.

Cabe recordar, por otra parte, respecto al art. 64 de la Constitución de 1931, la autorizada opinión de Pérez Serrano, que no oculta su «escepticismo» hablando del «temperamento vehemente y arrebatado» propio de España y de los diputados y ministros. Para este autor, que escri-

15 Vid. en castellano el comentario de BüHLER, 2010: 213 y ss. Una revisión completa de la historia y la práctica en el periodo de entreguerras del art. 54, así como de su influencia en la regulación actual, puede verse en BERTHOLD, 1997. Allí se atribuye la paternidad del voto de censura constructivo a Carlo Schmid, miembro de los «órganos constituyentes» (Verfassunskonvents von Herrenchiemse y Parlamentarischen Rat) de finales de los cuarenta, y a Ernst Fraenkel, que apuntó esta solución ya en escritos de 1932 (pp. 83 y ss.).

${ }^{16}$ Sobre este artículo vid. el comentario contenido en Pérez SERRANo, 1932: 239 y ss. 
be poco después de la aprobación de la norma suprema republicana, las correspondientes dimisiones vienen «sin formularse reproche o mostrarse desafecto notorio», en el caso de que «el voto recaído en algún asunto importante le resta autoridad o le deja sin el prestigio indispensable» ${ }^{17}$. El repaso que de la práctica en este periodo ha hecho, muchos años después, Vírgala ${ }^{18}$, demuestra que con unas Cortes fragmentadas y un jefe del Estado intervencionista poco podía hacer la norma citada para frenar la inestabilidad gubernamental.

Que en los dos supuestos, a estos débiles sistemas constitucionales les sustituyeran regímenes autoritarios/totalitarios fue la base para que, superadas dichas experiencias, se exploraran soluciones de reforzamiento del ejecutivo, sin excluir, por supuesto, el control de los respectivos Parlamentos. No hay que olvidar que estos deseos se enmarcaban en una tendencia muy consolidada y que tenía su origen en épocas anteriores: la que puede denominarse, haciendo quizás una síntesis injusta de su riqueza, «racionalización del parlamentarismo» o «parlamentarismo racionalizado» ${ }^{19}$.

La decisión clave fue la de hacer que quienes censuren al jefe del gobierno deban presentar una alternativa clara, de modo que no era suficiente con estar de acuerdo en derrocar aquél, sino, cosa bastante más difícil, los parlamentarios debían otorgar la investidura como canciller/presidente a su sucesor en el mismo acto ${ }^{20}$.

La inclusión de un candidato a la presidencia, que la ocupa si se aprueba la moción, es, por tanto, un elemento básico en ambos sistemas. Así, la Ley Fundamental de Bonn ${ }^{21}$ nos dice que: «El Bundestag sólo podrá plantear una moción de censura frente al canciller federal si elige [...] a un sucesor y solicita del presidente federal el relevo del canciller federal» (art. 67.1). En España la moción «habrá de incluir un candidato a la Presidencia del gobierno» (art. 113.2 CE), y si ésta se aprueba, «se entenderá investido de la confianza de la Cámara [...] El rey le nombrará presidente del gobierno» (art. 114.2 CE).

17 Ibid., 240.

18 Vírgala Foruria, 1988: 104 y ss.

19 El clásico sobre estos conceptos es MirKinE-Guetzévitch, 2011 (1933): esp. 43 y ss.

${ }^{20}$ Un repaso a los debates en el proceso constituyente alemán en donde surgió esa solución en SCHENKE, 1999: 3 y ss. En pp. 7 y ss. referencias a la historia del problema.

${ }^{21}$ Para entender la regulación en la LFB puede uno servirse de los diferentes comentarios al art. 67 de la misma. Hemos manejado los de Epping, 2010 (bibliografía de apoyo en pp. 1370-1371); SCHENKE, 1999 (bibliografía de apoyo en p. 75), y HeRzoG, 2008 (bibliografía en pp. 1 y 2). 
De este modo no habría ningún tipo de vacío de poder. Se pensaba, además, que, dado que no era fácil construir una alternativa seria, quedaría reforzada significativamente la posición de quien ejercía el cargo de canciller/presidente del gobierno, de modo que sólo pudiese ser sustituido por quien contara, a su vez, con amplios y sólidos apoyos en las Cámaras.

Otros detalles importantes, como la regla de que la moción debe ser presentada por un número significativo de diputados, que en Alemania es «una cuarta parte de los miembros del Bundestag o [...] un grupo parlamentario que englobe, al menos, una cuarta parte de los miembros del Bundestag» (art. 97 del Reglamento del Bundestag) y en España «al menos la décima parte» (art. 113.2 CE), contribuyen a dotar de un mínimo de seriedad al intento de derrocar el gobierno.

Ambas regulaciones establecen, asimismo, un periodo obligatorio de «enfriamiento» entre la presentación de la moción y la votación. En Alemania es de cuarenta y ocho horas (art. 67.2 LFB), y entre nosotros, que, al parecer, necesitamos poner más calma en el asunto - también porque está abierta la posibilidad de que aparezcan mociones alternativas en las dos primeras jornadas-, de cinco días (art. 113.3 CE).

En este repaso de la normativa queda por hacer una referencia a otro elemento de la misma que contribuye también al blindaje del canciller/ presidente del gobierno que se encuentre ejerciendo el cargo: se trata de la regla que exige que para desplazarlo hace falta una mayoría especialmente reforzada. En el caso alemán se habla de «mayoría de sus miembros» (art. 67.1 LFB), esto es, la llamada «mayoría de canciller», de acuerdo con lo previsto en el art. 121 LFB $^{22}$. En España, la moción de censura deberá ser adoptada por «mayoría absoluta» (art. 113.1 CE).

Se ha señalado repetidas veces la paradoja de que en este modo de acceder a la presidencia se debe contar con la mitad más uno de los parlamentarios, mientras que en los procedimientos normales de hacerse con la misma está prevista, tanto en Alemania (art. 63.4 LFB) como en España (art. 99.3 CE), la posibilidad de que al nuevo canciller/presidente del gobierno le baste con la mayoría simple.

Nos encontramos así que determinados jefes de gobierno (podríamos llamarles «minoritarios»), cuya investidura puede producirse por mecanis-

${ }^{22}$ Dice dicha norma que: «A los efectos de la presente Ley Fundamental, se considera mayoría de los miembros del Bundestag y de la Asamblea Federal, la mayoría del número legal de sus miembros». Es decir, añadimos nosotros, la mitad más uno de los mismos. Hay que recordar que, debido al peculiar sistema electoral alemán, este número legal varía en cada elección. 
mos relativamente sencillos, gozan de un excelente blindaje, pues quienes intenten sustituirlos necesitarán de unos apoyos más amplios.

Estamos hablando nada menos que de la mayoría absoluta de sus miembros, resultado que no es habitual que un partido consiga en los últimos tiempos en Alemania. Aunque en España se haya dado con cierta frecuencia, es difícil también que se construya con posterioridad a las elecciones. Entre nosotros, la perspectiva de que un líder político agrupe en torno suyo una mayoría absoluta para derrocar al presidente del gobierno parece remota.

\section{LAS PARADÓJICAS ENSEÑANZAS DE LA PRÁCTICA}

Repasadas las normas más significativas, es ahora imprescindible examinar cuál ha sido el funcionamiento en la práctica de este instrumento.

Resulta curioso, en primer lugar, que lo que puede caracterizar nuestra revisión es que el principal efecto de tan peculiares reglas consiste en que los actores políticos no osan lanzarse a una aventura como ésta a no ser que, tras las elecciones y la constitución de las Cámaras, se produzcan cambios significativos en los posicionamientos de grupos relevantes respecto a un determinado canciller/presidente del gobierno.

De hecho, si hemos realizado bien el repaso a la historia de la institución —en Alemania y en España, y en el nivel del Estado central- es preciso recordar que solamente una vez (Alemania, 1982) ha triunfado una moción de censura, y ello porque se dio una situación muy peculiar en la que estaban presentes en el Bundestag tres grandes fuerzas políticas (CDU/CSU, SPD y FPD), de las cuales una ocupaba el centro del espectro político y decidió dejar la coalición de gobierno de la que formaba parte hasta entonces con los socialistas para reconstruirla con los cristianodemócratas. Éste es el único supuesto en el que el mecanismo que estamos examinando provocó el cambio de canciller, y pronto veremos que con unas curiosas consecuencias.

Por otra parte, y como inmediatamente le pareció claro a todo el mundo que un cambio de gobierno mediante moción de censura sólo puede darse en circunstancias excepcionales, el instrumento se convirtió en otra cosa, sobre todo en el caso español. Y ello porque, en nuestra práctica, no se trata ya de derrocar al ejecutivo, sino de conseguir que la ciudadanía saque de ese proceso la impresión de que los grupos opositores tie- 
nen un programa alternativo de gobierno coherente y, sobre todo, un líder perfectamente capaz de ocupar la presidencia del mismo.

Desde el momento en que los debates, tal y como están regulados en el Reglamento del Congreso (arts. 175 a 179; especialmente art. 177.1), suponen que primero interviene un representante de los diputados que han presentado la moción criticando al ejecutivo todavía en el poder e, inmediatamente después, el candidato propuesto en la moción para ocupar la presidencia del gobierno, está claro que lo que se discute no son los posibles errores de quien está aún ejerciendo ese cargo, sino la capacidad del que encabeza la moción para sustituirlo. Además, sobre esto giran las intervenciones del resto de los grupos parlamentarios previstas en el art. 177.2 RC.

La práctica de las mociones de censura que en España han tenido lugar [1980, con Felipe González (PSOE) como candidato, y 1987, en la que el pretendiente era Antonio Hernández Mancha (AP)] demuestra que ocurre lo que acabamos de explicar. Es muy significativo que, en los dos casos, la iniciativa fracasara, si lo que se pretendía era cambiar de presidente del gobierno.

También lo es que, en el caso de la de 1980, Felipe González diera la imagen de que podía hacerse cargo de la presidencia y que el PSOE contaba con un programa de gobierno coherente. Esto contribuyó, en no poca medida, a la victoria socialista en las elecciones de octubre de 1982. Por el contrario, en 1987 el candidato no estuvo acertado, lo que provocó que, poco después, fuera sustituido en la presidencia de Alianza Popular.

Por tanto, en un caso, la moción, independientemente de que fracasara, sirvió para reforzar las posibilidades del candidato y de su partido de tener éxito mediante elecciones, por supuesto, en sus afanes de alcanzar el puesto que pretendían, mientras que, en el otro, tuvo como principal consecuencia demostrar que nos hallábamos ante una persona que no era la idónea para ocupar dicho cargo y un partido que debía mejorar su oferta política.

Todavía más curioso es lo que sucedió en Alemania la única vez que ha triunfado una moción de censura y, por tanto, se ha nombrado un nuevo canciller (recordemos que en $1982 \mathrm{Kohl}$ desbancó a Schmidt) ${ }^{23}$. Aunque nadie negó que, con la norma suprema en la mano, quien había alcanzado dicho cargo de aquella manera estaba plenamente legitimado para ejercer-

${ }^{23}$ El otro caso fue el fallido intento de Rainer Balzer (CDU/CSU) de desbancar a Willy Brandt (SPD) en 1972. 
lo, inmediatamente circuló la idea de que parecía necesario que se produjeran nuevas elecciones. Un proceso electoral que refrendara lo que, al fin y al cabo, algunos consideraron que era un cambio en el ejecutivo que procedía de algo así como un «golpe palaciego».

Dicha maniobra era perfectamente legítima y legal, en tanto en cuanto venía prevista en la Ley Fundamental de Bonn (recordemos que en el art. 67), pero había sido realizada al margen del electorado, y sobre ella debía pronunciarse éste por el método habitual: la llamada a las urnas. Hubo, por cierto, que recurrir a una falsa pérdida de confianza para lograr la disolución del Bundestag (art. 68 LFB) ${ }^{24}$, prohibida en otros casos.

Se trató de ver si los ciudadanos respaldaban la nueva coalición CDU/ CSU-FPD. Lo que se pretendía, como ha escrito Volker Epping, era añadir, «en el caso de canciller elegido a través de una moción de censura constructiva, a su legalidad constitucional una legitimidad procedente de las nuevas elecciones» ${ }^{25}$.

Vemos, por tanto, que, incluso en el raro caso de que la moción triunfe, parece obligado refrendar el cambio de jefe de gobierno con una Appellatio ad populum, lo que supone que el mecanismo, en realidad, vale solamente para tres cosas: a) frenar los intentos, no meditados, de derrocar al gobierno que pretendan utilizar «emboscadas» en las Cámaras y que no estén apoyados en una mayoría alternativa seria y cohesionada $; b$ ) intentar presentar con la máxima publicidad el programa de la oposición y demostrar que su líder puede ser canciller/presidente, y $c$ ) poner al frente del ejecutivo a un nuevo canciller/presidente que, en todo caso, deberá inmediatamente buscar el apoyo del pueblo soberano mediante unas nuevas elecciones.

La pregunta que surge inmediatamente es la que sigue: ¿está la moción de censura alcanzando los objetivos que pretendían cubrir los que introdujeron el mecanismo en Alemania y en España?

\section{LAS DUDAS SOBRE LA OPERATIVIDAD DE LA MOCIÓN DE CENSURA}

En los dos países no han faltado los críticos de la moción de censura. Destacó, en las constituyentes españolas, el senador de designación real,

24 Vid. sobre el asunto y la reacción del Tribunal Constitucional alemán los materiales citados supra en la nota 11.

25 Epping, 2010: 1720. Pueden verse, en el mismo sentido, Herzog, 2008: 16, y SCHENKE, 1999: 50. 
Carlos Ollero, que hablaba de que la misma «puede hacer imposible la responsabilidad política del gabinete». Sostenía, apoyándose en autores germanos (Böckenförde, Sontheimer, Hennis y Friesenhan), que en Alemania «no ha funcionado bien» ${ }^{26}$.

El estudio clásico en el país germano es el de Domes, que concluye afirmando que «el voto de censura constructivo quiere asegurar la estabilidad y la continuidad sin garantizar el cambio» ${ }^{27}$.

En España se resalta que no se han evitado, como es lógico, las crisis extraparlamentarias, que son las habituales en toda Europa ${ }^{28}$. Al fin y al cabo, la verdadera virtualidad de la moción de censura, que muestra «el contraste existente entre la finalidad original [...] y los verdaderos objetivos de su utilización práctica» ${ }^{29}$, ha sido la de ser «un excelente instrumento publicitario» ${ }^{30}$ que «permite a la oposición abrir un debate de política general» ${ }^{31}$. Además, se insiste, con toda razón, en que lo verdaderamente decisivo para la estabilidad han sido «las características del sistema de partidos» ${ }^{32}$.

No es tampoco de despreciar el dato de que en las reformas democratizadoras de los países del Este europeo, zona de influencia alemana, solamente Polonia (art. 158 de su Constitución) y Eslovenia (art. 116 de su norma suprema) han adoptado el modelo de la moción de censura con candidato alternativo. Casi todos tienen las reglas propias del parlamentarismo racionalizado (número mínimo de proponentes, enfriamiento, mayorías reforzadas, etc.), pero no el carácter «constructivo».

Que lo hiciera, en su momento, Papúa Nueva Guinea ${ }^{33}$ no añade demasiado al hecho de que se trata de un instrumento extraño, justificado por las peculiares situaciones de Alemania en 1949 y España en 1978, y sobre el que quizá se debiera volver con una perspectiva crítica, ya que lo único que parece conseguir es un reforzamiento extremo del ejecutivo frente a las posibles crisis parlamentarias.

26 Ollero, 1986: 177 y ss.

27 Domes, 1977: 62.

${ }^{28}$ Como concluye en su análisis comparado SÁNCHEZ DE Dios, 1992, para quien «las crisis de gobierno en los actuales regímenes parlamentarios han dejado de ser crisis fundamentalmente parlamentarias» (p. 369).

29 ElÍAs MÉNDEZ, 2005: 137.

30 Vírgala ForuRia, 1988: 341.

31 SÁNCHEZ DE Dios, 1992: 380.

32 Montero Gibert y García Morillo, 1984: 164.

33 Dato que tomo de Vírgala Foruria, 1988: 340. 
Es curioso, sin embargo, como en ambos países la solución se ha recibido en los niveles autonómico ${ }^{34}$ y municipal ${ }^{35}$, e incluso en ordenamientos de base asociativa en los que también se valoran sus aportaciones a la estabilidad de los respectivos presidentes ${ }^{36}$. Por otra parte, las recientes propuestas de reforma constitucional en Italia se pronuncian a favor de la importación de este modelo, hablando, como alternativa a la situación actual, de la «moción de censura constructiva, aprobada por mayoría absoluta» (Commissione, 2013: 24 y 25).

Cabe preguntarse si el reforzamiento del que hablábamos más arriba sigue siendo necesario o, teniendo en cuenta la evolución del sistema de partidos, debieran flexibilizarse las relaciones Parlamento-Gobierno, en aras de construir un régimen parlamentario algo menos rígido en el que los intentos de censurar al ejecutivo tengan mayores facilidades para que las críticas fundadas no se encuentren con barreras insuperables y no tengan que buscar otros canales de expresión.

En ese sentido se han pronunciado, últimamente, incluso algunos de los máximos responsables de que la moción de censura «constructiva» esté en la norma suprema española ${ }^{37}$, al decir que «no estoy seguro que hoy siga siendo necesaria y conveniente, pues tal vez el daño que a la democracia irroga un excesivo blindaje de los gobiernos sea mayor que el que podría ocasionar una menor estabilidad».

Parece, pues, que es conveniente entrar en un periodo de reflexión crítica sobre la figura. En el mismo habrá que tener en cuenta tanto la experiencia vivida hasta ahora como si la misma sigue siendo necesaria en el contexto constitucional (en Alemania y en España) de los inicios del siglo XXI.

34 Sobre estas derivaciones vid. ELÍAs MÉNDEZ, 2005: 97 y ss.

35 Vid. el art. 197 de la Ley Orgánica del Régimen Electoral General.

36 Por ejemplo, el art. 45 del Estatuto de la Federación Española de Rugby, que en su primer párrafo dispone: «La crítica a la gestión del presidente de la FER podrá ser ejercida mediante moción de censura. A tal efecto deberá ser propuesta por al menos un tercio de los miembros de la Asamblea y deberá llevar incluida un candidato a presidente de la FER». El art. 46 de la misma normativa exige para la aprobación de la misma «mayoría absoluta de la Asamblea».

37 Así se manifestó Francisco Rubio Llorente, que, como letrado de las Cortes, asesoró a la ponencia constitucional en el sentido de la recepción de las soluciones alemanas, y recientemente, en el discurso de recepción del doctorado bonoris causa por la Universidad de Valladolid, invita a revisar el problema. La cita está tomada de RuBio LLORENTE, 2013: 28. 
Ignacio Torres Muro La moción de censura constructiva. Una respuesta alemana...

\section{BIBLIOGRAFÍA}

BERTHOLD, L. (1997), «Das konstruktive Misstrauensvotum und seine Ursprünge in der Weimarer Staatsrechtslehre», Der Staat, núm. 36, pp. 81-94.

BüHLER, O. (2010), «Texto de la Constitución alemana de agosto de 1919 y comentario sistemático a sus preceptos», en W. JellineK, O. Bühler y C. Mortati, La Constitución de Weimar (texto de la Constitución alemana de 11 de agosto de 1919), Madrid, Tecnos.

Canales Aliende, M. (1983), «La jefatura del Estado durante la Segunda República», en AAVV, Actas del IV Symposium de Historia de la Administración, Madrid, Instituto Nacional de Administración Pública, pp. 109-124.

Commissione Per le Riforme Constituzionale (2013), Relazione finale (Bozza non correta), Roma, 17 de septiembre.

Cruz Villalón, P. (2011), «La Ley Fundamental de Bonn en la evolución constitucional española», en C. Hohmann-Dennhardt, R. Scholz y P. Cruz VillaLÓN, Las Constituciones alemana y española en su aniversario, Madrid, Centro de Estudios Políticos y Constitucionales, pp. 43-60 (hay versión alemana del texto en el mismo libro).

Domes, J. (1977), «Regierungskrisen in Bund und Ländern un di Funktion des konstruktiven Misstrauensvotums», en P. Haungs, Res Publica. Studien zum Verfassungswesen. Dolf Sternberger zum 70 Gegurtstag, München, Wilhelm Fink.

ElíAs MÉndez, C. (2005), La moción de censura en España y Alemania, Madrid, Congreso de los Diputados.

Epping, V. (2010), «Comentario al art. 67», en H. Mangoldt, F., Klein y Ch. StarcK (eds.), Kommentar zum Grundgesetz, vol. 2, Art. 20-82, München, Franz Vahlen.

Frosini, T. E. (ed.) (2004), Il Premierato nei Governi Parlamentari, Torino, Giappichelli.

GonzÁlez Encinar, J. J. (1988), «Das konstruktive Misstrauensvotum in Spanien», Zeitschrift für Parlamentsfragen, vol. 19, núm. 3, pp. 334-338.

Gruppo di Milano (1983), Verso una nuova Costituzione, Milano, Giuffré.

Herzog, R. (2008), «Comentario al art. 67», en Th. Maunz y G. Durig, Grundgesetz Kommentar, vol. V, Art. 54-85, München, C. H. Beck.

Hesse, K. (1995), Grundzüge des Verfassungsrechts der Bundesrepublik Deutschland, 20. ${ }^{a}$ ed., Heidelberg, C. F. Müller.

Gusy, C. (1997), Die Weimarer Reichsverfassung, Tübingen, Mohr Siebeck.

Leyland, P. (2012), The Constitution of de United Kingdom. A Contextual Analysis, 2. ${ }^{\text {a }}$ ed., Oxford-Portland, Hart.

Martínez Sempere, E. (1984), «La disolución del Bundestag...», Revista española de Derecho Constitucional, núm. 12, pp. 169-205. 
Ignacio Torres Muro La moción de censura constructiva. Una respuesta alemana...

- (1998), «La disolución del Parlamento en Gran Bretaña», Revista de Estudios Políticos núm. 101, pp. 131-158.

Mellado Prado, P. (1988), La responsabilidad política del gobierno en el ordenamiento español, Madrid, Congreso de los Diputados.

Mirkine-Guetzévitch, B. [2001 (1933)], Modernas tendencias del Derecho constitucional, Madrid, Reus.

Montero Gibert, J. R., y García Morillo, J. (1984), El control parlamentario, Madrid, Tecnos.

Moreno Luzón, J. (ed.) (2003), Alfonso XIII. Un político en el trono, Madrid, Marcial Pons.

Pasquino, G. F. (2012), «El parlamentarismo al estilo italiano», en J. Lanzaro (ed.), Presidencialismo y parlamentarismo. América Latina y Europa Meridional, Madrid, Centro de Estudios Políticos y Constitucionales, pp. 307-336.

Maunz, T., y Zippelius, R. (1991), Deutsches Staatsrecht, 28. ${ }^{a}$ ed., München, C. H. Beck.

Ollero, C. (1986), Derecho y teoría política en el proceso constituyente español, Madrid, Centro de Estudios Constitucionales.

Pegoraro, L. (1983), Il Governo in Parlamento, l'esperienza della V Repubblica francese, Padova, CEDAM.

Pérez Serrano, N. (1932), La Constitución española (9 de diciembre 1931). Antecedentes. Texto. Comentarios, 1. ' ed., Madrid, Revista de Derecho Privado.

Rubio Llorente, F. (2013), «Discurso de ingreso en el Ilustre Claustro de la Universidad de Valladolid», recogido en Universidad de Valladolid, Doctorado «Honoris Causa» del Excmo, Sr. D. Francisco Rubio Llorente, Valladolid.

Saiz Moreno, F. (1983), «Acerca de la disolución del Bundestag...», Revista Española de Derecho Constitucional, núm. 8, pp. 209-245.

SÁnchez de Dios, M. (1992), La moción de censura, Madrid, Congreso de los Diputados.

SimON YARZA, F. (2015), «La moción de censura: ¿constructiva u "obstructiva”?», REDC, año 35, núm. 103, pp. 87-109.

Schenke, W. R. (1999), «Comentario al art. 67», en R. Dolzer, K. Vogel y K. Grasshof (eds.) Bonner Kommentar zum Grundgesetz, t. 9, Arts. 62-73, Heidelberg, C. F. Müller.

Tomás Villarroya, J. (2012), Breve historia del constitucionalismo español, 12.. ed., Madrid, Centro de Estudios Políticos y Constitucionales.

Torres Muro, I. (2009), «Refrendo y Monarquía», Revista española de Derecho Constitucional, núm. 87, pp. 43-70.

Vírgala Foruria, E. (1988), La moción de censura en la Constitución española de 1978, Madrid, Centro de Estudios Constitucionales. 